\title{
Potential of Cross-infection of Colletotrichum Species Causing Anthracnose in Persimmon and Pepper
}

\author{
Hye-Ryoung Kim ${ }^{1}$, Tae Heon $\mathrm{Lim}^{2}$, Joohyung Kim${ }^{1}$, Young Ho Kim${ }^{3}$ and Heung Tae Kim${ }^{1 *}$ \\ 'Department of Plant Medicine, Chungbuk National University, Cheongiu 361-753, Korea \\ Research Institute of Agri-Bio Science, Samhoub, Co., Ltd. Sangiu 742-130, Korea \\ 'Department of Applied Biology and Chemistry, Seoul National University, Seou' 151-742, Korea \\ (Received on November 18, 2008; Accepted on Febriary 11. 2009)
}

Ninety isolates of Colletotrichum species from new persimmon tree twigs and 50 isolates from pepper plant fruits were isolated via single-spore isolation. Of the 140 isolates, 26 were examined for mycelial growth, carbendazim sensitivity, and ITS sequence. Four of the isolates from the persimmon trees, which were cultivated exclusively in an orchard, showed fast mycelial growth and sensitivity to carbendazim, while five of the pepper isolates showed slower mycelial growth and were resistant to the fungicide. However, 17 isolates from persimmon trees cultivated with pepper plants in the same orchard showed slow mycelial growth like the pepper isolates and they were sensitive to carbendazim like the persimmon isolates. ITS sequence analysis of these 27 isolates led to the identification of the 22 persimmon isolates as $C$. gloeosporioides and the five pepper isolates as C. acutatum. PCR with species-specific primers confirmed that the 90 isolates from persimmon were $C$. gloeosporioides whereas the $\mathbf{5 0}$ isolates from pepper were $C$. acutatum. The 90 persimmon isolates of $C$. gloeosporioides and 50 pepper isolates of $C$. acutatum were compared by a wound inoculation test to determine their capacity for host cross-infection. All of the $C$. acutatum isolates from pepper caused typical symptoms of anthracnose on the fruits of pepper plants and twigs of persimmon; they differed from the $C$. gloeosporioides isolates from persimmon, more than $\mathbf{9 0 \%}$ of which were able to infect only persimmon. Amplified fragment length polymorphism analysis revealed the existence of two groups (C. gloeosporioides and $C$. acutatum isolates group). At $80 \%$ genetic similarity, the $C$. gloeosporioides group was defined within four clusters, while the $C$. acutatum group was within three clusters. However, these clusterings were unrelated with the virulence of Colletotrichum species against pepper fruits.

Keywords: Colletotrichum acutatum, C. gloeosporioides, cross-infection, pepper, persimmon

\footnotetext{
* Corresponding author.

Phone) +82-43-261-2556, FAX) +82-43-271-4414

E-mail)htkim@cbnu.ac.kr
}

Morphological characteristics such as colony morphology, conidial shape, the presence or absence of setae and sclerotia, and the appressorium shape and size have been used to differentiate species in the genus Colletotrichum. Morphological traits, however, are highly variable among isolates and often subject to interpretation. Other characters, such as growth rate and sensitivity to benomyl, have been useful in differentiating between $C$, acutatum and $C$. gloeosporioides. Colletotrichum gloeosporioides is a ubiquitous, proliferate, and economically important pathogen that causes substantial yield losses due to fruit decay and vegetative tissue damage in a variety of plant species. Anthracnose, dieback, root rot, leaf spot, blossom rot, and seedling rot caused by C. gloeosporioides have been reported for a wide range of plants including avocado, almond, peach (Freeman et al., 1998), pepper (Park and Kim, 1992; Manadhar et al., 1995), citrus (Timmer et al., 1994), rubber tree (Brown and Soepena, 1994), and strawberry (Denoyes and Baudry, 1995). In comparison, C. acutatum affects many of the hosts that are susceptible to other Colletotrichum species (spp.). For example, strawberry plants may be infected by $C$. acutatum, C. gloeosporioides, or C. fragariae (Freeman and Katan, 1997; Gunnell and Gubler, 1992). Among citrus plants, whereas $C$. gloeosporioides is a common saprophyte and may cause postharvest anthracnose from preharvest quiescent infections, $C$. acutatum causes distinct diseases in different species, such as postbloom fruit drop and key lime anthracnose (Timmer et al., 1994). Interestingly, in Colletotrichum, multiple hosts can be affected by a single species, and multiple species can affect a single host.

A number of cross-infection studies have been conducted using $C$. gloeosporioides and C. acutatum, and a wide range of hosts (Mass and Howard, 1985; Alahakoon et al., 1994; Freeman et al., 1996). The various virulence of $C$. gloeosporioides isolated from several hosts was shown in other hosts (Quimio and Quimio, 1975; Freeman and Shabi, 1996). Quimio and Quimio (1975) found differences in the extert of virulence among 11 isolates of $C$. gloeosporioides cn green carabao mango. They also reported that isolates of C. gloeosporioides from mango, citrus, and 
papaya caused infections on the fruits of each of these hosts; however, there was variability in the degree of virulence. In fact, some isolates of $C$. gloeosporioides from mango did not infect banana or guava. Fagan (1979) was unable to cross-infect citrus flowers with an inoculum from senescent grapefruit leaves. Isolates of $C$. gloeosporioides from almond, apple, mango, and avocado inoculated into detached apple, avocado, almond, mango, and nectarine were shown to produce successful cross-infections (Freeman and Shabi, 1996). Furthermore, using conidial suspensions of isolates of $C$. acutatum from apple and peach, Freeman and Shabi (1996) inoculated detached nectarine, mango, avocado, almond, and apple fruits. All of the isolates produced lesions on the fruits, but it was necessary to wound the mango fruit to produce an infection. Peres et al. (2002) found that a $C$. acutatum isolate from strawberry produced lesions on wounded, detached avocado, guava, papaya, mango, and some fruits but not on banana. Isolates of $C$. acutatum from strawberry also produced lesions on wounded and unwounded fruits from pear, peach, nectarine, and apple (Freeman et al., 2001). Thus, it appears that Colletotrichum spp. have a broad host range and are relatively nonspecific. As many hosts susceptible to Colletotrichum spp. are cultivated worldwide, the losses in fields where mango, avocado, coffee, papaya, and citrus are grown in close proximity could be enormous (Freeman et al., 1998).

In Sangiu, Korea, there are several orchards where persimmon trees have been cultivated with pepper. Severe infections caused by anthracnose pathogens have been observed in both pepper and persimmon. In particular, persimmon anthracnose has had disastrous effects on the cultivation and production of persimmon (Lee et al., 2001). Kwon et al. (2000) reported the cultural characteristics of C. gloeosporioides, which causes persimmon anthracnose. In pepper anthracnose, the pathogen was identified as $C$. acutatum based on such characteristics as morphology, mycelial growth, benomyl sensitivity, and an analysis of the ITS region using molecular markers (Kim et al., 2008). Because crops such as pepper are often cultivated in close proximity to persimmon orchards in Sangju, Korea, it is important to determine whether these hosts can serve as inoculum sources for one another. In this study, we identified isolates obtained from persimmon based on their mycelial growth characteristics and carbendazim sensitivity, as well as a phylogenetic analysis of their ITS regions by PCR using species-specific primers. We also examined the virulence of each isolate on their respective hosts and their potential for cross-infection.

\section{Materials and Methods}

Isolation of the anthracnose pathogens. To obtain isolates through single-spore isolation, pieces of diseased persimmon twigs and fruits from pepper plants grown in persimmon orchards were surface-sterilized with $2 \%$ sodium hypochlorite for $1 \mathrm{~min}$ then washed twice with sterile distilled water (SDW) and placed in plastic containers $(30 \times 20 \times 10 \mathrm{~cm}, \mathrm{~W} \times \mathrm{L} \times \mathrm{H})$ lined with three paper towels soaked with $100 \mathrm{ml}$ of distilled water to maintain high humidity $(>95 \%, \mathrm{RH})$. After incubation, the spores produced on the pieces were harvested with SDW and washed with SDW twice. The spore concentration was adjusted to $1 \times 10^{6}$ conidia/ml and $50 \mu \mathrm{l}$ of the resulting suspension was spread on potato dextrose agar (PDA; Difco, Franklin Lakes, NJ, USA) supplemented with $300 \mu \mathrm{g} / \mathrm{ml}$ streptomycin. After 3 days of incubation at $25^{\circ} \mathrm{C}$, mycelial discs at the margins of small colonies were removed and subcultured. The isolates were maintained on PDA slants at $4^{\circ} \mathrm{C}$ until use.

Mycelial growth and carbendazim sensitivity. To measure mycelial growth, a 5-mm-diameter mycelial disc taken from the margin of a 7-day-old culture grown on PDA was inoculated in the center of PDA plates. The colony diameter of each isolate was measured daily for 1 week at $25^{\circ} \mathrm{C}$. To determine the carbendazim sensitivity, agar blocks were taken from the margins of the colonies grown on PDA at $25^{\circ} \mathrm{C}$ for 7 days and transferred to PDA plates containing the fungicide at $10 \mu \mathrm{g} / \mathrm{ml}$. Carbendazim (a.i. 95\%) was dissolved in dimethyl sulfoxide (DMSO) and added to the medium at the indicated concentration just before pouring into the Petri plates. DMSO alone was used as a control. The final concentration of DMSO was $<1 \%$ in all cases. After incubation at $25^{\circ} \mathrm{C}$ for 7 days, the colony diameter in each culture was evaluated on PDA with or without the fungicide. Three replicates were prepared for each isolate in both experiments.

Cross-infection test. Detached persimmon twigs and detached fruits from pepper plants were used to assess the pathogenicity of each isolate. The twigs and fruits were wounded with a pin and inoculated with a $5-\mu l$ drop of a conidial suspension adjusted to $5 \times 10^{5}$ conidia $/ \mathrm{ml}$. Conidia were harvested from cultures of two species of Colletotrichum incubated at $25^{\circ} \mathrm{C}$ for 10 days. The inoculated materials were placed in plastic containers $(30 \times 20 \times 10 \mathrm{~cm}$, $\mathrm{W} \times \mathrm{L} \times \mathrm{H}$ ) lined with three paper towels soaked with $100 \mathrm{ml}$ of SDW to maintain high humidity. Lesion length was measured after 10 days at $25^{\circ} \mathrm{C}$.

Genomic DNA Extraction. Total DNA was extracted from mycelia obtained from the PDA cultures after 10 days of growth at $25^{\circ} \mathrm{C}$. Aerial mycelia were harvested from the culture plates using a sterile transfer needle and placed in 
sterile $1.5-\mathrm{ml}$ microcentrifuge tubes containing $200 \mu \mathrm{l}$ of extraction buffer $(0.2 \mathrm{M}$ Tris- $\mathrm{HCl}, 0.25 \mathrm{M} \mathrm{NaCl}, 25 \mathrm{mM}$ EDTA, and 2\% SDS, pH 8.5). The tubes were placed in a boiling water bath for $5 \mathrm{~min}$ and then cooled to $25^{\circ} \mathrm{C}$. Then, $200 \mu \mathrm{l}$ of phenol that was equilibrated with extraction buffer $(v / v)$ and $200 \mu l$ of chloroform were added. The tubes were subsequently vortexed for $4 \mathrm{~min}$ and centrifuged at $13,000 \times \mathrm{g}$ for $5 \mathrm{~min}$. The supernatants were then transferred to fresh sterile $1.5-\mathrm{ml}$ tubes, and $200 \mu \mathrm{l}$ of chloroform was added; each mixture was then vortexed for $30 \mathrm{~s}$ and centrifuged at $13,000 \times \mathrm{g}$ for $15 \mathrm{~min}$. The supernatants were then extracted with $200 \mu \mathrm{l}$ of isopropanol and centrifuged at $13,000 \times \mathrm{g}$ for $15 \mathrm{~min}$. Next, the nucleic acid pellets were washed with $70 \%$ ethanol, air-dried for $15 \mathrm{~min}$, and resuspended in $50 \mu \mathrm{l}$ of TE buffer $(10 \mathrm{mM}$ Tris- $\mathrm{HCl}$ and $0.1 \mathrm{mM}$ EDTA, pH 8.5).

Sequencing of the ITS region. The primers ITS5 and ITS4-3 were used for amplification of the ITS region (Kang et al., 2005). Amplification was conducted in a total reaction volume of $25 \mu \mathrm{l}$ using a PCR kit (Bioneer Inc., Daejeon, Korea). The parameters used were as follows: a 2min hold at $95^{\circ} \mathrm{C}$ followed by 40 cycles of $1 \mathrm{~min}$ at $95^{\circ} \mathrm{C}, 1$ $\min$ at $55^{\circ} \mathrm{C}$, and $1 \mathrm{~min}$ at $72^{\circ} \mathrm{C}$ with a final extension for 5 $\min$ at $72^{\circ} \mathrm{C}$. The amplicons were column-purified using a PCR purification kit (Atman Biosciences, Uiwang, Korea) and cycle-sequenced by Eugentech Inc. (Taejon, Korea). The sequences were analyzed using both EditSeq and MegAlign (DNASTAR Inc., Madison, WI, USA).

PCR with species-specific primers for the identification of Colletotrichum spp. The primers CgInt (5'-GGC CTC CCG CCT CCG GGC GG-3') and Cal-3 (5'-CAG GGG AAG CCT CTC GCG GGC CT-3') were designed based on sequence similarities in the ITS1 region between $C$ gloeosporioides and C. acutatum (Mills et al., 1992; Kim et al., 2008). Cgint (specific for C. gloeosporioides) or Cal-3 (specific for $C$ acutatum) was used with the backward primer ITS4-3, which is specific for both $C$. gloeosporioides and C. acutatum. Each reaction mix $(30 \mu l)$ contained 50 ng of DNA, $1 \mathrm{M}$ each primer, and $15 \mathrm{ml}$ of PCR Master Mix (Promega, Madison, WI, USA). Amplification was conducted with at least three replicates as follows: one cycle of $4 \mathrm{~min}$ at $94^{\circ} \mathrm{C}$ followed by 30 cycles of $30 \mathrm{~s}$ at $94^{\circ} \mathrm{C}, 30 \mathrm{~s}$ at $65^{\circ} \mathrm{C}$, and $1 \mathrm{~min}$ at $72^{\circ} \mathrm{C}$ with a final $7-\mathrm{min}$ extension at $72^{\prime \prime} \mathrm{C}$. The products were then separated on $0.7 \%$ agarose gels.

Amplified fragment length polymorphism (AFLP) analysis. AFLP analysis was performed as described with modifications (Vos et al., 1995). About $1 \mu \mathrm{g}$ of genomic DNA was digested with EcoRI and MseI (New England
Biolabs UK Ltd., Hertfordshire, UK). The fragments were then ligated to double-stranded restriction site-specific adaptors using T4 DNA ligase (Takara, Shiga, Japan) and diluted 1:10 with TE buffer. Pre-selective PCR was performed using E00 (5'-GAC TGC GTA CCA ATT C-3') and M02 (5'-GAT GAG TCC TGA GTA AC-3') in a reaction mix $(25 \mu \mathrm{l})$ containing $0.5 \mu \mathrm{M}$ each primer, $1 \mathrm{X}$ Taq buffer ( $2 \mathrm{mM}$ Tris- $\mathrm{HCl}, 10 \mathrm{mM} \mathrm{KCl}, 10 \mu \mathrm{M}$ EDTA, $0.1 \mathrm{mM}$ DTT, $0.05 \%$ NP-40, $0.05 \%$ Tween $20,5 \%$ glycerol, and $\left.2 \mathrm{mM} \mathrm{MgCl}_{2}\right), 0.2 \mathrm{mM}$ dNTPs, $1 \mathrm{U}$ of Taq polymerase (Enzynomics, Daejeon, Korea), and $2 \mu 1$ of diluted DNA. PCR was performed as follows: $5 \mathrm{~min}$ at $95^{\circ} \mathrm{C}$ followed by 20 cycles of $30 \mathrm{~s}$ at $95^{\circ} \mathrm{C}, 1 \mathrm{~min}$ at $56^{\circ} \mathrm{C}$, and $1 \mathrm{~min}$ at $72^{\circ} \mathrm{C}$ with a final 7 -min extension at $72^{\circ}$. For selective PCR, $2 \mu 1$ of a 1:10 dilution of each pre-selective product was amplified using E42 (5'-GAC TGC GTA CCA ATT CTC-3') and M213 (5'-GAT GAG TCC TGA GTA ACA G-3') in a 25- $\mu \mathrm{l}$ volume with the following parameters: pre-denaturation at $95^{\circ} \mathrm{C}$ for $5 \mathrm{~min}$ followed by 12 cycles of denaturation at $95^{\circ} \mathrm{C}$ for $30 \mathrm{~s}$, a $0.7^{\circ} \mathrm{C}$ step-wise reduction in the annealing temperature from $65^{\circ} \mathrm{C}$ for $30 \mathrm{~s}$, and extension at $72^{\circ} \mathrm{C}$ for $1 \mathrm{~min}$, plus an additional 23 cycles with annealing at $56^{\circ} \mathrm{C}$ for $30 \mathrm{~s}$. The AFLP fragments were size-fractionated in $4 \%$ polyacrylamide sequencing gels and visualized using the Silverstar" Staining System (Bioneer Inc.). The AFLP profiles were analyzed using GelCompar II (Applied Maths BVBA, Sint-Martens-Latem, Belgium). A phylogenetic tree was constructed using Pearson's correlation coefficient and cluster analysis by the unweighted pair group method with arithmetic averages (UPGMA).

\section{Results}

Characteristics of the persimmon and pepper isolates. Mycelial growth, carbendazim sensitivity, and virulence were investigated using isolates obtained from persimmon and pepper. The persimmon isolates were divided into two groups: the group 1 isolates including 5 isolates from PER 1 to PER5 in Table 1 were taken from diseased twigs in an orchard where only persimmon trees were cultivated, while the group 2 isolates including 17 isolates from PPF 1 to PPF17 were taken from an orchard in which persimmon trees and pepper plants were cultivated simultaneously. As shown in Table 1, none of the isolates obtained from persimmon showed virulence in the pepper fruits or resistance to carbendazim. However, the isolates obtained from diseased pepper fruits grown in a persimmon orchard showed high virulence on pepper fruits and were able to grow on PDA containing $10 \mu \mathrm{g} / \mathrm{ml}$ carbendazim. In terms of the mycelial growth of the persimmon isolates, a difference was detected between the group 1 and group 2 isolates. All of the group 2 isolates grew less than $50.0 \mathrm{~mm}$ during 7 days of incubation 
Table 1. Virulence on pepper fruits, mycelial growth on PDA, and carbendazim sensitivity of Colletotrichum isolates from persimmon and pepper, respectively

\begin{tabular}{|c|c|c|c|c|}
\hline Isolate $^{a}$ & Host Plant & $\begin{array}{l}\text { Virulence }^{b} \text { on } \\
\text { Pepper Fruit } \\
\text { (mm) }\end{array}$ & $\begin{array}{l}\text { Mycelial } \\
\text { Growth }^{c} \\
(\mathrm{~mm})\end{array}$ & $\begin{array}{l}\text { Carbendazim } \\
\text { Sensitivity } \\
\text { (mm) }\end{array}$ \\
\hline PER I & Persimmon & 0.0 & 65.6 & 0.0 \\
\hline PER 2 & Persimmon & 0.0 & 64.3 & 0.0 \\
\hline PER 3 & Persimmon & 0.0 & 61.2 & 0.0 \\
\hline PER 4 & Persimmon & 0.0 & 62.6 & 0.0 \\
\hline PER 5 & Persimmon & 0.0 & 64.5 & 0.0 \\
\hline PPF 1 & Persimmon & 0.0 & 40.9 & 0.0 \\
\hline PPF 2 & Persimmon & 0.0 & 44.9 & 0.0 \\
\hline PPF 3 & Persimmon & 0.0 & 45.4 & 0.0 \\
\hline PPF 4 & Persimmon & 0.0 & 43.3 & 0.0 \\
\hline PPF 5 & Persimmon & 0.0 & 41.1 & 0.0 \\
\hline PPF 6 & Persimmon & 0.0 & 42.3 & 0.0 \\
\hline PPF 7 & Persimmon & 0.0 & 47.3 & 0.0 \\
\hline PPF 8 & Persimmon & 0.0 & 43.4 & 0.0 \\
\hline PPF 9 & Persimmon & 0.0 & 39.5 & 0.0 \\
\hline PPF 10 & Persimmon & 0.0 & 43.6 & 0.0 \\
\hline PPF 11 & Persimmon & 0.0 & 48.7 & 0.0 \\
\hline PPF 12 & Persimmon & 0.0 & 37.4 & 0.0 \\
\hline PPF 13 & Persimmon & 0.0 & 42.8 & 0.0 \\
\hline PPF 14 & Persimmon & 0.0 & 39.7 & 0.0 \\
\hline PPF 15 & Persimmon & 0.0 & 38.4 & 0.0 \\
\hline PPF 16 & Persimmon & 0.0 & 50.6 & 0.0 \\
\hline PPF 17 & Persimmon & 0.0 & 43.7 & 0.0 \\
\hline PEP 1 & Pepper & 15.9 & 37.5 & 18.3 \\
\hline PEP 2 & Pepper & 15.7 & 40.1 & 14.5 \\
\hline PEP 3 & Pepper & 27.6 & 42.7 & 15.1 \\
\hline PEP 4 & Pepper & 16.8 & 41.5 & 16.2 \\
\hline
\end{tabular}

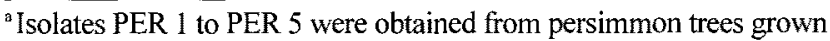
in orchards without pepper, while isolates PPF 1 to PPF 17 were from persimmon trees grown in orchards where persimmon and pepper were cultivated simultaneously. Isolates PEP 1 to PEP 4 were obtained from pepper plants grown in persimmon orchards.

${ }^{6}$ Pepper fruits were inoculated with a conidial suspension $\left(5 \times 10^{5}\right.$ conidia/ml) by dropping $5 \mu \mathrm{l}$ of the suspension onto sites of wounding. The lesion lengths were measured after 10 days of incubation at high humidity in a plastic box at $25^{\circ} \mathrm{C}$.

'The colony diameter of the Colletotrichum species on PDA was investigated after incubation for 7 days at $25^{\circ} \mathrm{C}$.

${ }^{d}$ After incubation at $25^{\circ} \mathrm{C}$ for 7 days, the colony diameter in each culture was evaluated on PDA containing $10 \mu \mathrm{g} / \mathrm{ml}$ carbendazim.

on PDA at $25^{\circ} \mathrm{C}$, except for isolate PPF 16 , which showed the same mycelial growth tendency as the pepper isolates. In comparison, the group 1 isolates grew faster than the pepper and group 2 isolates, with a mean growth of $63.6 \mathrm{~mm}$.

ITS sequence analysis and species-specific PCR. The phylogenetic relationships among the Colletotrichum isolates were inferred by UPGMA analysis of the nucleotide sequences of the amplified ITS regions (Fig. 1). According to our results, the Colletotrichum isolates fit well into two distinct groups: C. acutatum and C. gloeosporioides. While all of the pepper isolates possessed the ITS sequence of $C$. acutatum, all of the persimmon isolates belonged to the $C$. gloeosporioides group. A portion of the ITS region was amplified from the pepper and persimmon Colletotrichum isolates using primers designed for $C$. acutatum (Cal-1) or C. gloeosporioides (CgInt). The primer sequences were derived from regions showing species-specific variation in the ITS region. Using the C. acutatum-specific primer Ca11 and the reverse primer ITS4-3, a 499-bp fragment was amplified from each of the pepper isolates PEP 1, PEP 2, and PEP 3 in Fig. 2. Using CgInt and ITS4-3, a 466-bp fragment was amplified from each of the persimmon isolates, including the 16 persimmon isolates in group 2 (PPF 1-PPF 16) and the five persimmon isolates in group 1 (PER 1-PER 5). Furthermore, it was confirmed that the others of the 90 persimmon isolates were $C$. gloeosporioides while the others of 50 pepper isolates were $C$. acutatum.

Comparison of the cross-infection potential. The $\mathrm{Col}$ letotrichum spp. analyzed in this study corresponded to 140 isolates, 50 of which were $C$. acutatum isolates from pepper and 90 of which were $C$. gloeosporioides isolates from persimmon. Of the 90 isolates from persimmon, $45 / 46$ in group 1 and 43/44 in group 2 produced typical symptoms of anthracnose on detached persimmon twigs (Table 2). In contrast, among the pepper fruits, only four of the C. gloeosporioides isolates from group 1 and six C. gloeosporioides isolates from group 2 showed symptoms of anthracnose with a mean lesion size of $2.6 \mathrm{~mm}$ on pepper fruits. Nonetheless, out of the 50 pepper isolates identified as $C$. acutatum, 48 showed strong virulence, producing typical symptoms of anthracnose on pepper fruits. Moreover, all of the isolates produced symptoms of anthracnose on new persimmon twigs, with reduced virulence compared to the persimmon isolates. The mean lesion length caused by the pepper isolates was $9.0 \mathrm{~mm}$ on new persimmon twigs, compared to 13.0 and $12.4 \mathrm{~mm}$ for the persimmon isolates in groups 1 and 2 , respectively.

AFLP analysis. Using AFLP, 140 isolates of Colletotrichum spp. from persimmon and pepper were analyzed for genetic diversity, and a dendrogram was constructed by the UPGMA method (Fig. 3). At 80\% genetic similarity, seven clusters were defined and gathered into two groups: the C. acutatum isolates and the $C$. gloeosporioides isolates. Minimum genetic similarity occurred between the two groups at 0.009 . The $C$. acutatum isolate group was divided into three clusters. Cluster B contained only C. acutatum PEP 14, which showed the greatest virulence on twigs of persim- 


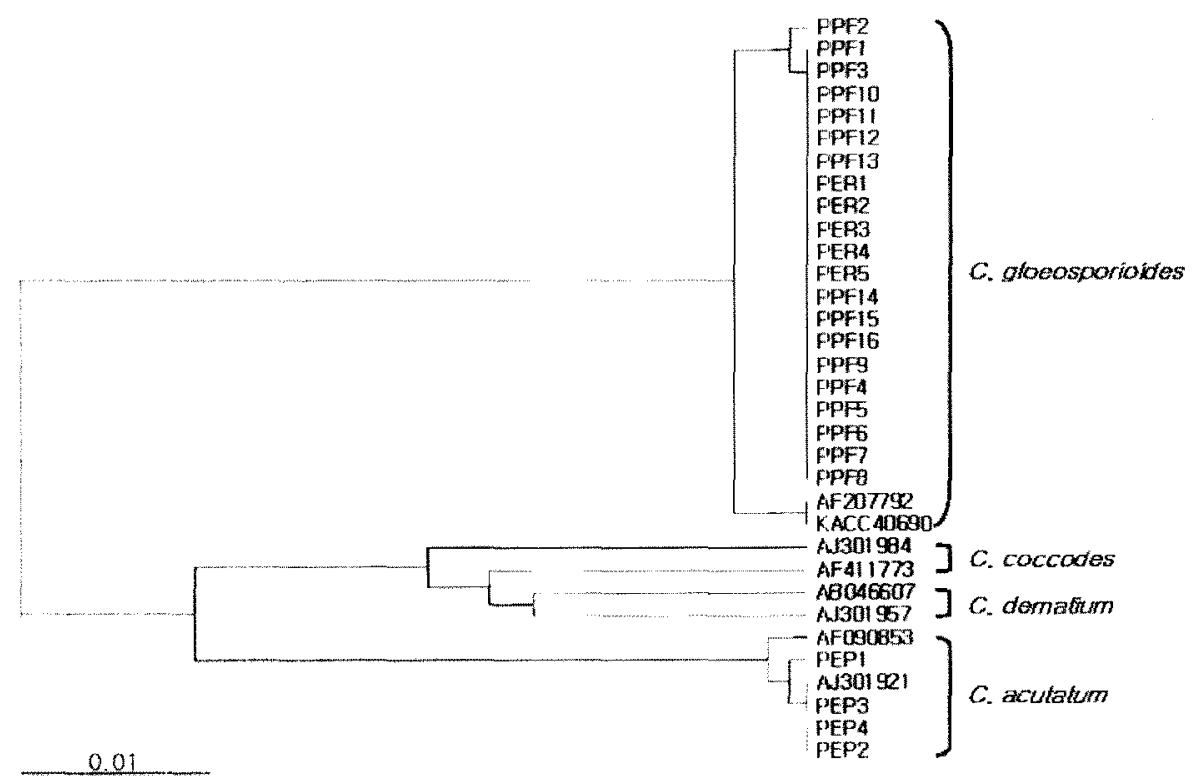

Fig. 1. An ITS-based phylogenetic tree of the Colletotrichun isolates. The tree was constructed using cluster analysis with the unweighted pair group method with arithmetic averages (UPGMA). The branch orders were similar in all tree-construction approaches used. The scale bar indicates an estimated $1 \%$ sequence divergence.

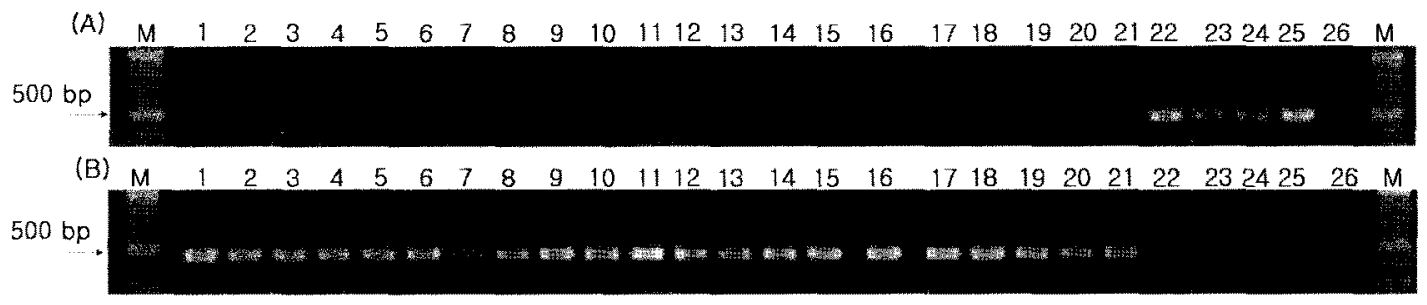

Fig. 2. Amplification of a species-specific fragment from fungal DNA using the primers Cal-3 and ITS4-3 for Colletotrichm acuatum (A) or Cglnt and ITS4-3 for C. gloeosporioides (B). Lane: M, size marker; 1, PER 1; 2, PER 2; 3, PER 3; 4, PER 4; 5, PER 5; 6, PPF 1; 7, PPF $2 ; 8$, PPF $3 ; 9$, PPF $4 ; 10$, PPF $5 ; 11$, PPF $6 ; 12$, PPF $7 ; 13$, PPF $8 ; 14$, PPF $9 ; 15$, PPF 10; 16, PPF 11; 17, PPF 12; 18, PPF 13; 19, PPF 14;20, PPF 15;21, PPF 16;22, PEP 1;23, PEP 2; 24, PEP 3; 25, PEP 4; 26, C. gloeosporioides KACC40690.

Table 2. Isolates of Colletotrichum species summarized according to host plant and their virulence on persimmon and pepper

\begin{tabular}{|c|c|c|c|c|}
\hline \multirow{2}{*}{$\begin{array}{l}\text { Host Used } \\
\text { in this Study }\end{array}$} & \multirow{2}{*}{$\begin{array}{c}\text { Species, } \\
\text { Collection Host }\end{array}$} & \multicolumn{3}{|c|}{ Number of Isolates } \\
\hline & & Pathogenic & Nonpathogenic & Total \\
\hline \multirow[t]{3}{*}{ Pepper } & C. gloeosporioides, persimmon only ${ }^{\mathrm{h}}$ & 4 & 42 & 45 \\
\hline & C. gloeosporioides, persimmon with pepper & 6 & 38 & 44 \\
\hline & C. acutatum, pepper with persimmon ${ }^{d}$ & 48 & 2 & 50 \\
\hline \multirow[t]{3}{*}{ Persimmon } & C. gloeosporioides, persimmon only & 45 & 1 & 46 \\
\hline & C. gloeosporioides, persimmon with pepper & 43 & 1 & 44 \\
\hline & C. acutatum, pepper with persimmon & 50 & 0 & 50 \\
\hline
\end{tabular}

Persimmon and pepper were used as the host plants for the virulence test of all Colletotrichum isolates.

"Obtained from diseased persimmon twigs in an orchard where persimmon was cultivated alone.

'Obtained from diseased persimmon twigs in an orchard where persimmon was cultivated with pepper plants.

"Obtained from diseased pepper fruits in an orchard where persimmon was cultivated with pepper plants.

"Persimmon twigs and pepper fruits were wounded by a pin and inoculated by dropping $5 \mu \mathrm{l}$ of a conidial suspension adjusted to $5 \times 10^{\circ}$ conidia/ $\mathrm{ml}$ onto the wound. Lesion length was measured after incubation of the inoculated materials at $25^{\circ} \mathrm{C}$ for 10 days in plastic containers $(30 \times 20 \times 10$ $\mathrm{cm}, \mathrm{W} \times \mathrm{L} \times \mathrm{H}$ ) lined with three paper towels soaked with $100 \mathrm{ml}$ of distilled water to maintain high humidity. 


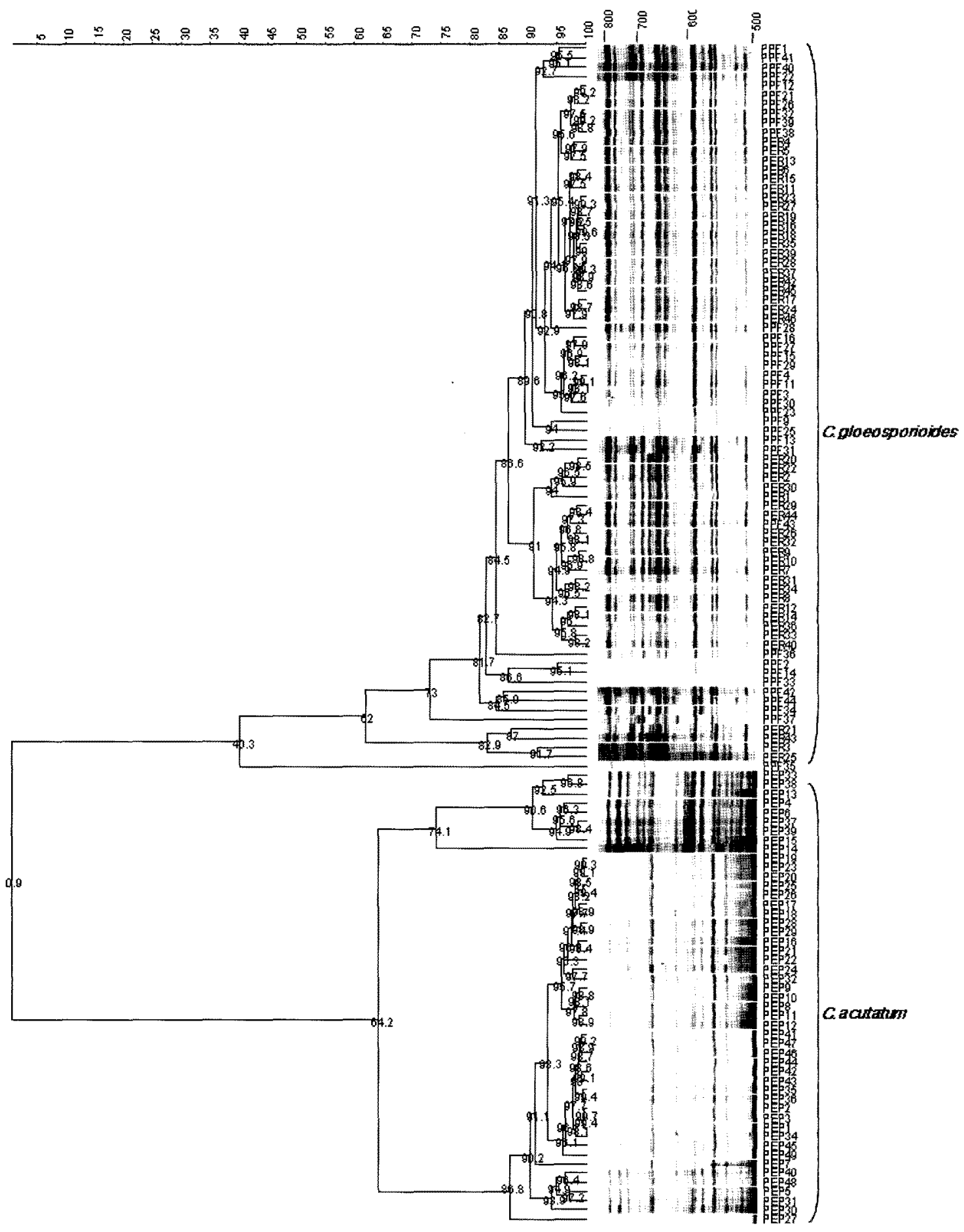

Fig. 3. Consensus dendrogram generated by 1,000 bootstrap reiterations using GelCompar II (Applied Maths BVBA) to analyze the AFLP data for 140 Colletotrichum species isolates. The dendrogram was constructed using Pearson's correlation coefficient and cluster analysis with UPGMA.

mon among the $50 \mathrm{C}$. acutatum isolates. Clusters $\mathrm{A}$ and $\mathrm{C}$ contained 41 and 8 isolates of $C$. acutatum, respectively. The C. gloeosporioides isolate group was divided into four clusters. Clusters D and F each contained a single isolate: C. gloeosporioides PPF 35 and PPF 37, respectively. Four isolates, C. gloeosporioides PER 3, 21, 25, and 43, were included in cluster E. Cluster G, which included 84 isolates of C. gloeosporioides, was subdivided into nine clusters at $90 \%$ genetic similarity. 


\section{Discussion}

The Colletotrichum spp. identified in this study were divided into two groups: $C$. gloeosporioides and $C$. actutatum. All of the $C$. gloeosporioides isolates were obtained from persimmon, while those of $C$. acutatum were obtained from pepper. The Colletotrichum spp. were separated according to their host plant preferences. Although we were unable to detect cross-infection by the Colletotrichum spp. between persimmon and pepper in the field, it was inferred that the Colletotrichum spp. could attack either host plant due to low host specificity. In reality, our cross-infection results indicated some variation in the host preference of the $C$. gloeosporioides and $C$. acutatum isolates from persimmon and pepper. Whereas the $C$. gloeosporioides isolates were much more pathogenic on persimmon than on pepper, the c. acutatum isolates were able to infect both hosts. In addition, there was a clear distinction between the $C$. gloeosporioides and $C$. acutatum isolates from persimmon and pepper based on the sequences of their ITS regions and AFL.P data. However, isolates of C. gloeosporioides were not divided into different two groups in AFLP data, as one was from the orchard where only persimmon trees were cultivated and the other was from the orchard with both of crops such as persimmon and pepper.

As shown in Table 2, while 88 isolates of C. gloeosporioides were able to produce symptoms on their original host, but only 10 isolates were able to do on pepper. Several previous reports have also noted the variation in virulence of C. gloeosporioides isolates (Darvas et al., 1987; Freeman and Shabi, 1996). These differences may be ascribed to adaptations of the pathogen to its host, allowing it to become more virulent and to overcome the host's defense mechanisms (Alahakoon et al., 1994). These adaptations may be highly host-specific, such that the ability of the pathogen to infect other hosts is eliminated. In contrast, the C. acutatum isolates used in this study appeared to be pathogenic toward both persimmon and pepper. It was previously reported that the causative pathogen of pepper anthracnose in Korea was C. acutatum (Kim et al., 2008); moreover, $C$. acutatum has been shown to have a broad host range and be relatively nonspecific (Peres et al., 2005). In research conducted in Israel, the inoculation of isolates of C. cututum from anemone and strawberry into each host resulted in typical symptoms of anthracnose on both host plants (Freeman et al., 2000). In contrast, although isolates of $C$. acutatum were recovered from pepper, eggplant, tomato, bean, and weeds of the genera Vicia and Conyza, disease symptoms occurred only in strawberry (Freeman et al., 2001). Thus, C. acutatum was reported to parasitize and cause disease on other hosts, or, alternatively, to survive on other crops or on weeds without producing symptoms. Than et al. (2008) presented the cross-infection potential of $C$. aculatum isolates from strawberry to fruits of the pepper $\mathrm{cv}$. Bangchang, which are susceptible to anthracnose caused by $C$. acutatum.

In the orchards of Sangiu, Korea, most farmers cultivate pepper plants between their persimmon trees. Based on our results, although $C$. gloeosporioides from persimmon was not able to infect pepper, $C$. acutatum isolated from pepper could infect not only pepper but also persimmon. This suggests that in the persimmon orchards of Sangiu, C. acutatum may cross-infect both pepper and persimmon plants.

Farmers could use benzimidazole fungicides to control persimmon anthracnose. Use of these fungicides might play a role in selection in persimmon orchards. Benzimidazole fungicides are largely effective against anthracnose, except in pepper (Lim and Choi, 2006; Kim et al., 2007). Even in our in vitro experiments, all of the C. gloeosporioides isolates appeared to be sensitive to carbendazim at $10 \mu \mathrm{g} / \mathrm{ml}$, although the $C$. acutatum isolates were resistant to the fungicide as reported by Kim et al. (2007). Mycelial growth and conidia production are routinely used to assess the field fitness of plant pathogens (Sacristán and Garcia-Arenal, 2008). Although the mycelial growth of $C$. gloesporioides was reported to be faster than that of $C$. acutatum, conidia production was lower in $C$. gloeosporioides than in $C$. acutatum (Agostini et al., 1992; Berstein et al., 1995; Liyanage et al., 1992; Kim et al., 2008). Thus, it was suggested that $C$. acutatum be considered as a major pathogen in persimmon orchards, where two species of Colletotrichum, namely $C$. gloeosporioides and $C$. acutatum, are distributed, due to its strong resistance to carbendazim and conidia production.

Our cross-infection analysis was conducted in the laboratory using detached and wounded twigs and fruits. Despite the significant potential for cross-infection on the detached materials used in this study, it remains to be determined whether isolates from a different host pose a serious threa: in the field, since our inoculation studies were carried out under optimum conditions for infection by the pathogen. There is currently no proof that the crossinfection of different hosts by $C$. acutatum actually occurs under field conditions; thus, additional studies are required.

\section{Acknowledgment}

This work was supported by a grant from BioGreen 21 Program, Rural Development Administration, Republic of Korea.

\section{References}

Agostini, J. P., Timmer, L. W. and Mitchell, D. J. 1992. Morpho- 
logical and pathological characteristics of strains of Colletotrichum gloeosporioides from citrus. Phytopathology 82:1377-1382.

Alahakoon, P. W., Brown, A. E. and Sreenivasaprasad, S. 1994. Cross-infection potential of genetic groups of Colletotrichum gloeosporioides on tropical fruits. Physiol. Mol. Plant Pathol. 44:93-103.

Berstein, B., Zehr, E. I., Dean, R. A. and Shabi, E. 1995. Characteristics of Colletotrichum from peach, apple, pecan, and other hosts. Plant Dis. 79:478-482.

Brown, A. E. and Soepena, H. 1994. Pathogenicity of Colletotrichum acutatum and Colletotrichum gloeosporioides on leaves of Hevea spp. Mycol. Res. 98:264-266.

Darvas, J. M., Kotzé, J. M. and Wehner, F. C. 1987. Pathogenicity of fungi causing pre- and postharvest diseases of avocado fruits. Phytophylactica 19:489-493.

Denoyes, B. and Baudry, A. 1995. Species identification and pathogenicity study of French Colletotrichum strains isolated from strawberry using morphological and cultural characteristics. Phytopathology 85:53-57.

Fagan, H. J. 1979. Postbloom fruit drop, a new disease of citrus associated with a form of Colletotrichum gloeosporioides. Ann. Appl. Biol. 91:13-20.

Freeman, S., Horowitz, S. and Sharon, A. 2001. Pathogenic and nonpathogenic lifestyles in Colletotrichum acutatum from strawberry and other plants. Phytopathology 91:986-992.

Freeman, S. and Katan, T. 1997. Identification of Colletotrichum species responsible for anthracnose and root necrosis of strawberry in Israel. Phytopathology 87:516-521.

Freeman, S., Katan, T. and Shabi, E. 1996. Characterization of Colletotrichum gloeosporioides isolates from avocado and almond fruits with molecular and pathogenicity tests. Appl. Environ. Microbiol. 62:1014-1020.

Freeman, S., Katan, T. and Shabi, E. 1998. Characterization of Colletotrichum species responsible for anthracnose disease of various fruits. Plant Dis. 82:596-605.

Freeman, S. and Shabi, E. 1996. Cross-infection of subtropical and temperate fruits by Colletotrichum species from various hosts. Physiol. Mol. Plant Pathol. 49:395-404.

Freeman, S., Shabi, E. and Katan, T. 2000. Characterization of Colletotrichum acutatum causing anthracnose of anemone (Anemone coronaria L.). Appl. Environ. Microbiol. 66:5267-5272.

Gunnell, P. S. and Gubler, W. D. 1992. Taxonomy and morphology of Colletotrichum species pathogenic to strawberry. Mycologia 84:157-165.

Kang, B. K., Min, J. Y., Kim, Y. S., Park, S. W., Van Bach, N. and Kim, H. T. 2005. Semi-selective medium for monitoring Colletotrichum acutatum causing pepper anthracnose in the field. Res. Plant Dis. 11:21-27.

Kim, J., Park, S. Choi, W., Lee, Y. and Kim, H. T. 2008. Characterization of Colletotrichum isolates causing anthracnose or pepper in Korea. Plant Pathol. J. 24:17-23.

Kim, Y-S., Min, J. Y., Kang, B. K., Van Bach, N., Choi, W. B.,
Park, E. W. and Kim, H. T. 2007. Analyses of the less benzimidazole-sensitivity of the isolates of Colletotrichum spp. causing anthracnose in pepper and strawberry. Plant Pathol. $J$. 23:187-192.

Kwon, J-H., Kang, S-W. and Park, C-S. 2000. Cultural characteristics of Colletotrichum gloeosporioides causing anthracnose of persimmon. Plant Dis. Res. 6:48-50 (in Korean).

Lee, D. W., Lee, G. C., Lee, S. W., Park, C-G, Choo, H. Y. and Shin, C-H. 2001. Survey on pest management practice and scheme of increasing income in sweet persimmon farms in Korea (in Korean). Korean J. Pest. Sci. 5:45-49.

Lim, T. H. and Choi, Y-H. 2006. Response of several fungicides of Colletotrichum gloeosporioides isolates obtained from persimmon in Sanju. Res. Plant Dis. 12:99-102.

Liyanage, H. D., McMillan, R. T. and Kistler, H. C. 1992. Two genetically distinct populations of Colletotrichum gloeosporioides from citrus. Phytopathology 82:1371-1376.

Manandhar, J. B., Hartman, G. L. and Wang, T. C. 1995. Anthracnose development of pepper fruits inoculated with Colletotrichum gloeosporioides. Plant Dis. 79:380-383.

Mass, J. L. and Howard, C. M. 1985. Variation of several anthracnose fungi in virulence to strawberry and apple. Plant Dis. 69:164-166.

Mills, P. R., Sreenivasaprasad, S. and Brown, A. E. 1992. Detection and differentiation of Collettorichum gloeosporioides isolates using PCR. FEMS Microbiol. Lett. 98:137-143.

Park, K. S. and Kim, C. H. 1992. Identification, distribution and etiological characteristics of anthracnose fungi of red pepper in Korea. Korean J. Plant Pathol. 8:61-69.

Peres, N. A., Kuramae, E. E., Dias, M. S. C. and de Souza, N. L. 2002. Identification of Colletotrichum species. Affecting fruit harvest in Brazil. J. Phytopathol. 150:128-134.

Peres, N. A., Timmer, L. W., Adaskaveg, L. E. and Correll, J. C. 2005. Lifestyles of Colletotrichum acutatum. Plant Dis. 89: 784-796

Quimio, T. H. and Quimio, A. J. 1975. Notes of Philippine grape and guava anthracnose. Plant Dis. Rept. 59:221-224.

Sacristán, S. and García-Arenal, F. 2008. The evolution of virulence and pathogenicity in plant pathogen populations. Mol. Plant Pathol. 9:369-384.

Than, P. P., Jeewon, R., Hyde, K. D., Pongsupasamit, S., Mongkolporn, O. and Taylor, P. W. J. 2008. Characterization and pathogenicity of Colletotrichum species associated with anthracnose on chilli (Capsicum spp.) in Thailand. Plant Pathol. 57:562-572.

Timmer, L. W., Agostini, J. P., Zitko, S. E. and Zulfiquar, M. 1994. Postbloom fruit drop, an increasingly prevalent disease of citrus in the Americas. Plant Dis. 78:329-334.

Vos, P., Hogers, R., Bleeker, M., Reijans, M., Vandelee, T., Hornes, M., Frijters, A., Pot, J., Peleman, J., Kuiper, M., Zabeau, M., 1995. AFLP-A new technique for DNA-fingerprinting. Nucleic Acids Res. 23:4407-4414. 